\title{
Precipitación de uranio a partir de licores alcalinos
}

\author{
Precipitation of uranium from \\ alkaline liqueurs
}

Ivana Orozco ${ }^{1}$, Marina Romero ${ }^{1}$, Rodolfo Lara ${ }^{1}$, Vanesa Bazan ${ }^{2}$

\footnotetext{
${ }^{1}$ Instituto de Investigaciones Mineras, Facultad de Ingeniería, Universidad Nacional de San Juan. Av. Libertador General San Martin 1109 Oeste. San Juan, San Juan, Argentina.

${ }^{2}$ CONICET-Instituto de Investigaciones Mineras, Facultad de Ingeniería, Universidad Nacional de San Juan. Av. Libertador General San Martin 1109 Oeste. San Juan, San Juan,Argentina.

e-mail: iorozco@unsj.edu.ar,mromero@unsj.edu.ar,rflara@unsj.edu.ar,bazan@unsj.edu.ar
}

\section{RESUMEN}

En Argentina existen minerales de uranio con abundante contenido de materia orgánica, conjuntamente variedad en cantidad de sulfuros y carbonatos que actúan como cemento o sellando diaclasas. Estos últimos minerales, modifican directamente la elección del proceso metalúrgico de lixiviación para la concentración de uranio. El proceso seleccionado más benéfico, resulta ser la lixiviación alcalina. Una vez realizada la lixiviación alcalina del mineral de uranio, mediante el uso del agente lixiviante como es el $\mathrm{Na}_{2} \mathrm{CO}_{3} / \mathrm{NaHCO}_{3}$ en presencia de agente oxidantes, se logra llegar al estado hexavalente del uranio como $\mathrm{U}_{3} \mathrm{O}_{8}$. En este trabajo de investigación se plantea el diseño experimental a nivel de laboratorio, con el fin de obtener la precipitación de uranio a partir de licores de lixiviación alcalina mediante dos mecanismos diferentes de precipitación. El primero utiliza un agentes oxidantes fuertes como el peróxido de hidrogeno en exceso. Estequiométricamente, se utilizó $0,9 \mathrm{~g}$ de $\mathrm{H}_{2} \mathrm{O}_{2}$, obteniendo la precipitación de uranio como $\mathrm{UO}_{2} * 2 \mathrm{H}_{2} \mathrm{O}$. Las variables de trabajo son condiciones de $\mathrm{pH}$ débilmente ácidas, para valores comprendidos entre $2.5,4$ hasta 5 y tiempos de reacción con exposición del mineral de $1,2,3$ y 4 hrs. Las temperaturas de trabajo son de $25^{\circ} \mathrm{C}, 60{ }^{\circ} \mathrm{C}$ y $80{ }^{\circ} \mathrm{C}$ para una velocidad de agitación constante de $400 \mathrm{rpm}$. En el segundo mecanismo se adiciona un agente precipitante como es el $\mathrm{NaF}$ en medio alcalino, sin modificación del $\mathrm{pH}$ de los licores lixiviados, generando un compuesto insoluble como es el $\mathrm{NaUF}_{6}$. Para ello se trabajó con distintas concentraciones del agente precipitante, velocidad de agitación y tiempos de precipitación. Sin embargo el mecanismo de mejor repuesta fue el primero, donde los valores de recuperación de uranio óptimos fueron encontrados para condiciones de trabajo de pH: 2,5; temperatura de $60{ }^{\circ} \mathrm{C}$ y tiempos de reacción superiores a 3 h hasta 4 h.

Palabras clave: uranio, precipitación, lixiviación alcalina.

\section{ABSTRACT}

In Argentina there is uranium with abundant organic matter and variety in quantity of sulfides and carbonates which act as cement or sealing joints. The latter minerals modify directly the choice of metal leaching process for the concentration of uranium. The most beneficial selected process turns out to be the alkaline leaching. Once this process has been finished, using the lixiviante agent such as $\mathrm{Na}_{2} \mathrm{CO}_{3} \mathrm{NaHCO}_{3}$ in presence of oxidizing agent, the hexavalent state of uranium as $\mathrm{U}_{3} \mathrm{O}_{8}$ has been achieved. In this research the experimental design is proposed at the laboratory level, in order to obtain the precipitation of uranium from alkaline leach liquors using two different precipitation mechanisms. One of them uses a strong oxidizing agent such as excessive hydrogen peroxide. Stoichiometrically, $0.9 \mathrm{~g}$ of $\mathrm{H}_{2} \mathrm{O}_{2}$ were used, obtaining the uranium precipitation as $\mathrm{UO}_{2} * 2 \mathrm{H}_{2} \mathrm{O}$. The working variables are lightly acidic $\mathrm{pH}$ values, for values between $2.5,4$ to 5 and reaction times with mineral exposure of $1,2,3$ and 4 hours. Working temperatures are $25{ }^{\circ} \mathrm{C}, 60{ }^{\circ} \mathrm{C}$ and 80 ${ }^{\circ} \mathrm{C}$ for a constant stirring speed of $400 \mathrm{rpm}$. In the other mechanism, a precipitating agent such as $\mathrm{NaF}$ in alkaline medium is added, without modifying the $\mathrm{pH}$ of leached liquors, generating an insoluble compound such as $\mathrm{NaUF}_{6}$. For this purpose we worked with different: concentrations of the precipitating agent, stirring speed and precipitation times. However, the first mechanism proved to be the best. Uranium recovery values 
were found for working conditions of $\mathrm{pH}$ : 2.5 ; temperature of $60^{\circ} \mathrm{C}$ and reaction times higher than $3 \mathrm{~h}$ until 4h.

Keywords: uranium precipitation, alkaline leaching

\section{INTRODUCCIÓN}

Actualmente la lixiviación alcalina está adquiriendo un mayor desarrollo. Debido a su comportamiento poco corrosivo y no contaminante, ha desarrollado un papel importante cuando se aplica lixiviaciones in situ. Los reactivos alcalinos utilizados, según MILLER y STANNARD [1] son $\mathrm{Na}_{2} \mathrm{CO}_{3} / \mathrm{NaHCO}_{3}$, por ende para condiciones de $\mathrm{pH}$ alcalino el uranio se encuentra estable en solución. Las reacciones químicas preponderantes en condiciones alcalinas son las ecuaciones 1 y 2 respectivamente.

$$
\begin{aligned}
& 2 \mathrm{UO}_{2}+\mathrm{O}_{2} \rightarrow 2 \mathrm{UO}_{3} \\
& \mathrm{UO}_{3}+\mathrm{Na}_{2} \mathrm{CO}_{3}+2 \mathrm{NaHCO}_{3} \rightarrow \mathrm{Na}_{4} \mathrm{UO}_{2}\left(\mathrm{CO}_{3}\right)_{3}+\mathrm{H}_{2} \mathrm{O}
\end{aligned}
$$

Según se observa en las ecuaciones químicas 1 y 2 el uranio lixiviado que se encuentra en los licores forma un complejo estable como un carbonato de sodio uranilo $\mathrm{Na}_{4} \mathrm{UO}_{2}\left(\mathrm{CO}_{3}\right)_{3}$ en solución alcalina. Como lo que se busca es alterar ese equilibrio para obtener la precipitación de uranio. Uno de los posibles agentes precipitantes según SMIRNOV et al [2] puede ser el peróxido de hidrógeno $\left(\mathrm{H}_{2} \mathrm{O}_{2}\right)$ en exceso estequiométrico en condiciones de pH débilmente ácidos.

Si se analiza el reactivo $\mathrm{H}_{2} \mathrm{O}_{2}$ en exceso estequiométrico la ecuación química que representa el mecanismo de precipitación es ejemplificada por la ecuación 3. La reacción química 3 se logra cuando se obtienen las condiciones de $\mathrm{pH}$ débilmente acida, mediante la adición de un ácido oxidante como el $\mathrm{HNO}_{3}$. La concentración de ácido a agregar se establece según lo indica la estequiometria de la reacción. Una segunda condición es la concentración en exceso de $\mathrm{H}_{2} \mathrm{O}_{2}$, se determina con el análisis estequeométrico de la siguiente reacción, mostrada en MORAIS et al [3].

$\mathrm{Na}_{4} \mathrm{UO}_{2}\left(\mathrm{CO}_{3}\right)_{3}+4 \mathrm{H}_{3} \mathrm{O}^{+}+\mathrm{H}_{2} \mathrm{O}_{2} \rightarrow \mathrm{UO}_{4} 2 \mathrm{H}_{2} \mathrm{O}+3 \mathrm{CO}_{2}+5 \mathrm{H}_{2} \mathrm{O}$

Es importante considerar la influencia del pH para obtener la mejor recuperación de $\mathrm{UO}_{4} \cdot 2 \mathrm{H}_{2} \mathrm{O}$. Sin embargo el parámetro más destacable e importante además del pH es la concentración en exceso de $\mathrm{H}_{2} \mathrm{O}_{2}$.

El proceso de precipitación es bastante complejo y queda mucho por entender de él. Esencialmente consiste en convertir el catión básico $\mathrm{UO}_{2}{ }^{2+}$ en un precipitado insoluble que puede contener una mezcla de compuestos: óxidos hidratados de uranio, sulfatos básicos y uranatos.

Los licores alcalinos típicamente contienen carbonatos de uranilo. Antes de la precipitación de los iones uranilo, los iones carbonato deben ser destruidos es por ello que se trabaja en pH levemente acido. Un ácido (usualmente $\mathrm{HCl}$ o $\mathrm{HNO}_{3}$ ) se agrega a la solución concentrada de carbonatos para destruir el carbonato para producir dióxido de carbono; el dióxido de carbono se ventea. Una vez que los carbonatos han sido destruidos, la solución acidificada se neutraliza con un álcali o se trata con peróxido de hidrógeno para precipitar los compuestos uraníferos, según se muestra en la ecuación 3.

El segundo mecanismo en estudio es el que utiliza un reactivo precipitante como el Fluoruro de sodio $(\mathrm{NaF})$ o el Fluoruro de sodio ácido (NaHF) en medio alcalino sin modificación del pH de los licores lixiviados, generando un compuesto insoluble como es el $\mathrm{NaUF}_{6}$. Para ello se trabajó con distintas condiciones de precipitación, tales como: concentración del agente precipitante, tiempos y velocidad de agitación. La reacción química se representa a continuación:

$$
\mathrm{Na}_{2} \mathrm{UO}_{2}\left(\mathrm{CO}_{3}\right)_{2(\mathrm{aq})}+6 \mathrm{NaF}_{(\mathrm{s})}=\mathrm{NaUF}_{6(\mathrm{~s})}+2 \mathrm{CO}_{2}+5 \mathrm{Na}
$$

\section{MATERIALES Y MÉTODOS}

\subsection{Lixiviación alcalina}

La muestra sólida de cabeza fue caracterizada mineralógica y químicamente previa al proceso de lixiviación alcalina fue secada y molida a malla - 200\# para su posterior análisis en el laboratorio químico. La metodología aplicada en la cuantificación química de $\mathrm{U}, \mathrm{Fe}, \mathrm{Al}, \mathrm{Na}, \mathrm{K}, \mathrm{Mo}, \mathrm{V}, \mathrm{Mn}, \mathrm{Ca}$ y Mg, consistió en una disgregación total de la muestra a analizar, realizando un ataque multiácido $\left(\mathrm{HF}, \mathrm{HCl} ; \mathrm{HNO}_{3} ; \mathrm{HClO}_{4}\right)$ y la cuantificación de analitos se llevó a cabo por Espectrometría de Emisión por Plasma Inductivamente Acoplado 
(ICP - OES), en un equipo Perkin Elmen 7300 DV. Los difractogramas de rayos X fueron obtenidos usando un difractómetro Rigaku Geigerflex operado a $30 \mathrm{kV}$ y $25 \mathrm{~mA}$, empleando una radiación $\mathrm{Cu} \mathrm{K} \alpha(\lambda=1,5405$ $\mathrm{nm}$ ), usando $\mathrm{NaCl}$ y cuarzo como estándares de calibración externos, con una velocidad de $20 \mathrm{~min}^{-1}$ en el rango $2 \theta$ comprendido entre 5-80.

La microcopia se realizó mediante el uso de Lupa binocular de 10x de las 3 clases determinadas. Con microscopio electrónico de barrido ESEM (FEI Quanta 200).

Con los resultados obtenidos de la caracterización química y mineralógica la muestra solida de cabeza se trató mediante lixiviación alcalina. Se utilizaron los siguientes reactivos lixiviantes $\mathrm{Na}_{2} \mathrm{CO}_{3}$ y $\mathrm{NaHCO}_{3}$. La relación entre ambos reactivos fue de $100 \mathrm{~kg} \mathrm{Na} \mathrm{CO}_{3} / \mathrm{t}$ de mineral y $50 \mathrm{~kg} \mathrm{NaHCO} / \mathrm{t}$ de mineral.

\subsection{Precipitación de uranio}

Los licores de uranio obtenidos de la lixiviación alcalina continúan el proceso metalúrgico mediante la precipitación en Bach con agitación y variación de temperatura para diferentes tiempos de contacto. Las variables operacionales consideradas fueron concentración del agente precipitante $\left(\mathrm{H}_{2} \mathrm{O}_{2}\right), \mathrm{pH}$, tiempos y temperaturas.

Para la etapa de precipitación se utilizó reactivos como $\mathrm{H}_{2} \mathrm{O}_{2}$, para distintas condiciones de pH. Los rangos de $\mathrm{pH}$ se encuentran comprendidos entre 2,5; 4 y 5. Los tiempos de reacción con exposición del mineral de $1,2,3$ y 4 hs. Las temperaturas de trabajo son $25^{\circ} \mathrm{C}, 60^{\circ} \mathrm{C}$ y $80^{\circ} \mathrm{C}$. La concentración de $\mathrm{H}_{2} \mathrm{O}_{2}$ fueron de 0,3 hasta $0,9 \mathrm{~g}$ de $\mathrm{H}_{2} \mathrm{O}_{2}\left(30 \%(\mathrm{p} / \mathrm{p})\right.$, densidad $\left.1,45 \mathrm{~g} / \mathrm{cm}^{3}\right)$.

Inicialmente se acidifica con $\mathrm{HNO}_{3}$ a temperatura ambiente y posterior a ello se agrega el reactivo oxidante en exceso estequiométrico de $\mathrm{H}_{2} \mathrm{O}_{2}$. Una vez condicionada la muestra se comienza a realizar el calentamiento para las temperaturas citadas. La regulación del $\mathrm{pH}$ se realiza con $\mathrm{NaOH}$ o $\mathrm{KOH}$.

El segundo mecanismo es utilizar $\mathrm{NaF}$ en solución para las concentraciones de $0.25 \mathrm{M}, 0.5 \mathrm{M}$ y $1 \mathrm{M}$; para cada una de las soluciones mencionadas se trabajó sin agitación Los tiempos de trabajo fueron hasta 24 hrs. Se analizaron cada uno de los productos obtenidos cuantificando la concentración de uranio existente en la solución. Las condiciones óptimas se obtuvieron considerando la menor concentración de uranio en solución en conjunto con un balance de masa. Una vez obtenida la condición mencionada anteriormente se modificaron la velocidad de agitación para la concentración constante $0.5 \mathrm{M}$ de $\mathrm{NaF}$, ellas fueron: sin agitación, $100 \mathrm{rpm}$ y $200 \mathrm{rpm}$.

\subsection{Caracterización de las muestras precipitadas}

Los licores resultantes de la precipitación del uranio fueron cuantificados para los analitos de U, Fe, Al, Na, $\mathrm{K}, \mathrm{Mo}, \mathrm{V}, \mathrm{Mn}, \mathrm{Ca}$ y Mg por medio de la técnica de ICP - OES, en un equipo Perkin Elmen $7300 \mathrm{DV}$.

\section{RESULTADOS}

\subsection{Análisis Mineralógicos}

Los resultados del análisis mineralógico de la muestra solida de cabeza se presentan en las Figura 1 y 2 , que en general la muestra está compuesta mayormente por pechblenda (variedad impura de uraninita) y otros sulfuros asociados a la mineralización, como pirita. La pechblenda aparece generalmente en forma masiva, diseminada en granos en la matriz de la arenisca o como relleno en microfracturas de pirita o material carbonáceo. Exhibe texturas coloformes en bandas concéntricas asociadas a un fracturamiento radial. La pirita es el sulfuro predominante en la arenisca, con cristales dispersos en la matriz de formas subhedrales-anhedrales y también como agregados framboidales. 

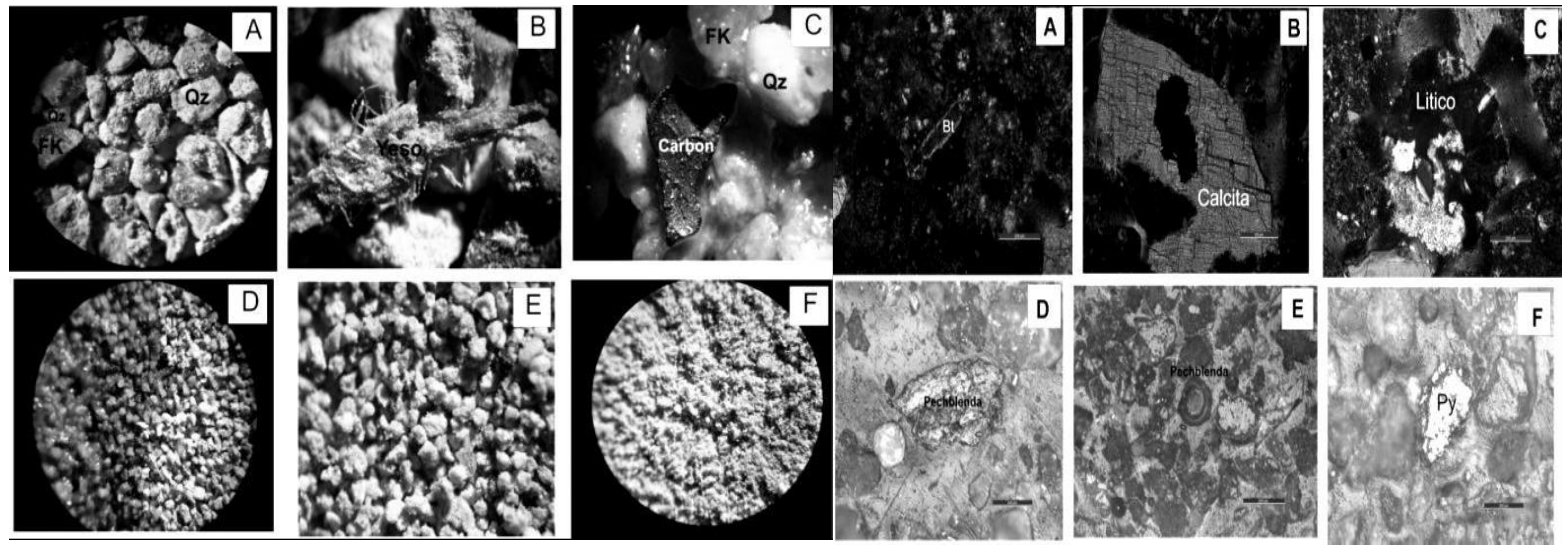

Figura 1: Microscopia con lupa. Especificaciones en texto.

Figura 2: Microscopia electrónica. Especificaciones en texto.

En la Figura 1, donde la Fotografía A: Imagen correspondiente a la clase [+30-100] donde pueden reconocerse: fragmentos de cuarzo $(\mathrm{Qz})$, feldespato potásico $(\mathrm{FK})$ con una cubierta de carbonatos. Fotografía B: Imagen de la clase [+30-100] de un mineral de yeso $\left(\mathrm{CaSO}_{4}\right)$ con textura fibrosa. Fotografía C: Imagen de la misma clase anterior, correspondiente a un fragmento de carbón mineral, cuarzo (Qz) y Feldespato potásico (FK). Fotografía D: Imagen correspondiente a la clase [+100-400], donde se visualiza el contacto de reacción entre $\mathrm{HCl}$ puro y parte de la muestra sin atacar. Fotografía E: Vista de los fragmentos minerales correspondiente a la clase [+400-400], todos ellos envueltos de una cubierta de carbonatos. Fotografía F: Corresponde a la clase -400 con un tamaño partícula de $38 \mu \mathrm{m}$, de igual manera cubierto de material carbonatico.

Para el análisis con difracción de rayos x según se observa en la Figura 3, la muestra original donde se identifica óxidos de uranio, cuarzo, calcita y sulfatos no se observan por estar presentes en concentraciones menores al $5 \%$.

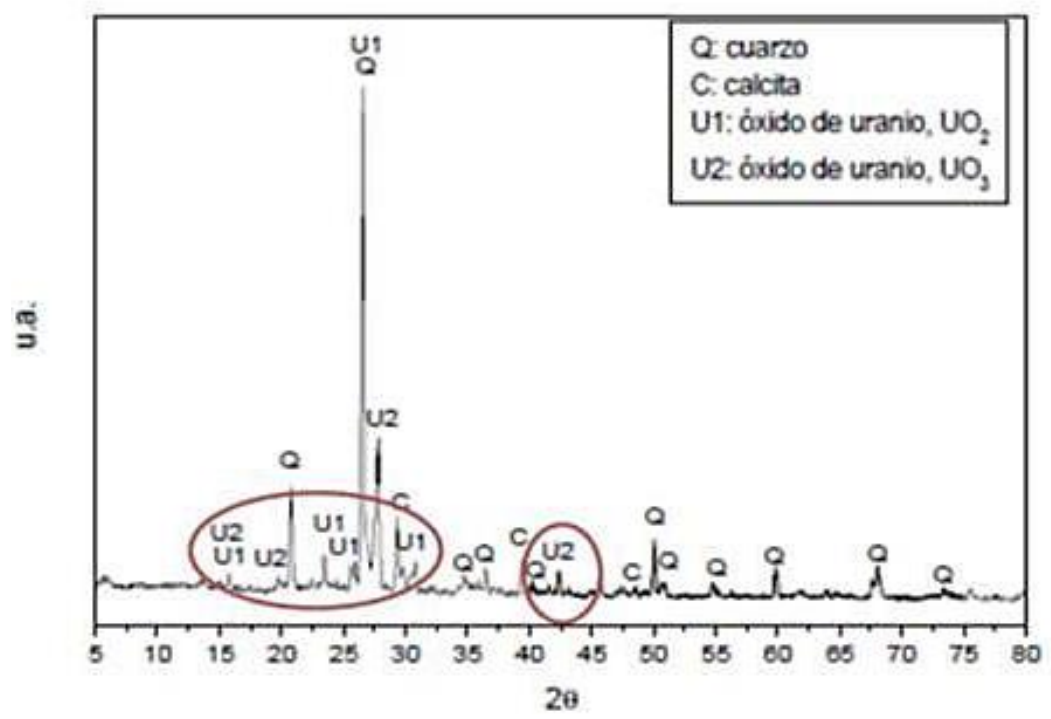

Figura 3: Difracción de Rayos X.

\subsection{Análisis Químico}

La composición química de las muestra de cabeza se pueden observar en la Tabla 1. La muestra original a lixiviar y posterior precipitación de uranio presenta las siguientes características químicas, expresadas en la Tabla 1. 
Tabla 1: Análisis químico de muestra de cabeza

\begin{tabular}{|c|c|c|c|c|c|c|c|c|c|c|c|}
\hline $\mathbf{U}_{\mathbf{3}} \mathbf{O}_{\mathbf{8}}$ & $\mathbf{F e}$ & $\mathbf{N a}$ & $\mathbf{K}$ & $\mathbf{M o}$ & $\mathbf{A l}$ & $\mathbf{V}$ & $\mathbf{M n}$ & $\mathbf{C a}$ & $\mathbf{M g}$ & P.a. $\mathbf{1 0 5}^{\mathbf{}} \mathbf{C}$ & Sol. Sol. \\
\hline $\boldsymbol{\mu g} / \mathbf{g}$ & $\boldsymbol{\mu g} / \mathbf{g}$ & $\boldsymbol{\mu g} / \mathbf{g}$ & $\boldsymbol{\mu g} / \mathbf{g}$ & $\boldsymbol{\mu g} / \mathbf{g}$ & $\boldsymbol{\mu g} / \mathbf{g}$ & $\boldsymbol{\mu g} / \mathbf{g}$ & $\boldsymbol{\mu g} / \mathbf{g}$ & $\boldsymbol{\mu g} / \mathbf{g}$ & $\boldsymbol{\mu g} / \mathbf{g}$ & $\boldsymbol{\%}$ & $\boldsymbol{\%}$ \\
\hline $\mathbf{4 0 7 . 2 5}$ & 100040 & 149 & 290 & 37 & 543 & 95,9 & 302 & 28020 & 5884 & 1,9 & 12,1 \\
\hline
\end{tabular}

-Muestra cabeza: muestra virgen, sin lixiviar

-P.a.: Perdidas de agua

-Sol. Sol: Sólidos solubles

\subsection{Análisis de variables operativas}

Se estudia el comportamiento del uranio frente a distintas condiciones operacionales en temperatura y tiempos para la etapa de precipitación. Para ello, se utilizó reactivos como $\mathrm{H}_{2} \mathrm{O}_{2}$ en exceso 0,3 a $0,9 \mathrm{~g}$. Los rangos de $\mathrm{pH}$ se encuentran comprendidos entre 2,5; 4 y 5 . Los tiempos de reacción con exposición del mineral fueron de $1,2,3$ y 4 hs. Las temperaturas de trabajo son $25^{\circ} \mathrm{C}, 60^{\circ} \mathrm{C}$ y $80^{\circ} \mathrm{C}$. Se busca encontrar la óptima concentración en exceso de $\mathrm{H}_{2} \mathrm{O}_{2}$ para la cual se obtiene la mayor recuperación de uranio, con la estructura química de $\mathrm{UO}_{4} 2 \mathrm{H}_{2} \mathrm{O}$.

En las Figuras 4 se muestran los ensayos de precipitación para cada una de las condiciones mencionadas. Es importante dejar claro que para las condiciones de 4 y 5 el comportamiento de las muestras es muy parecido, motivo por lo cual la información era relevante y no se mostró los gráficos para esas condiciones.

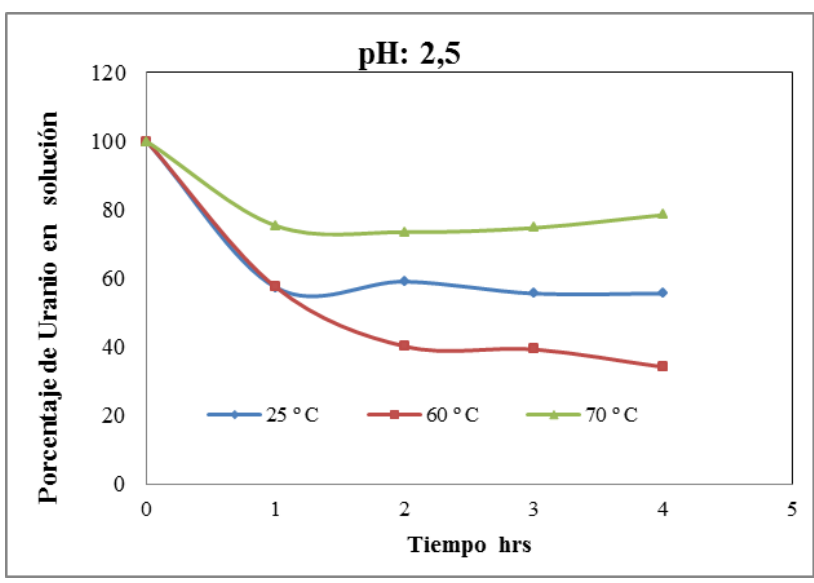

Figura 4: Ensayos de precipitación a pH: 2,5 para $25^{\circ} \mathrm{C}-60{ }^{\circ} \mathrm{C}$ y $80{ }^{\circ} \mathrm{C}$.

El segundo mecanismo de precipitación muestra los siguientes resultados para 0,25 M, 0,5M y $1 \mathrm{M}$ de $\mathrm{NaF}$ sin agitación para condiciones alcalinas de pH: 11,5, tiempos de precipitación de $24 \mathrm{hrs}$. Se selecciona la solución de $\mathrm{NaF}$ 0,5M para la cual se obtuvo la menor concentración de uranio en solución.

Para la concentración de $\mathrm{NaF}$ constante de $0,5 \mathrm{M}$ se modificaron las condiciones de agitación como: sin agitación, $100 \mathrm{rpm}$ y $200 \mathrm{rpm}$. En las Figuras 5 y 6 se muestran los ensayos de precipitación para cada una de las condiciones mencionadas. 


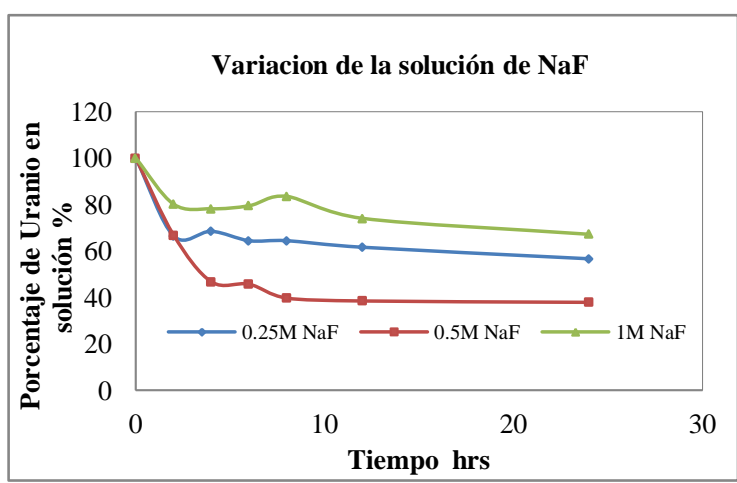

Figura 5: Ensayos de precipitación con variación de la concentración de NaF sin agitación.

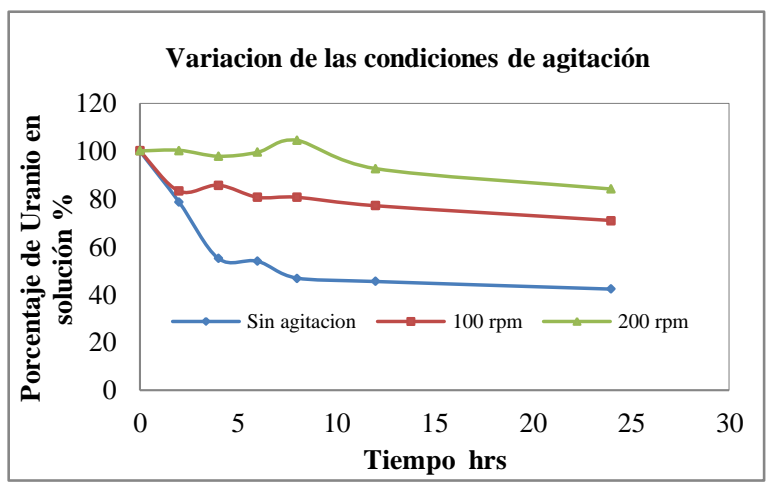

Figura 6: Ensayos de precipitación con variación de la velocidad de agitación para $0,5 \mathrm{M} \mathrm{NaF}$.

\section{DISCUSIONES}

Analizando los gráficos, se puede decir que para la condición de $\mathrm{pH}$ : 2,5 a las temperaturas de $25^{\circ} \mathrm{C}, 60^{\circ} \mathrm{C}$ y $80{ }^{\circ} \mathrm{C}$ la curva que mejor respuesta ha tenido es la obtenida a $60^{\circ} \mathrm{C}$ y para el tiempo de 3 a $4 \mathrm{hrs}$ se alcanza la estabilidad en la curva, motivo por el cual la precipitación del uranio ya no continua. En cambio para las temperaturas de $25^{\circ} \mathrm{C}$ y $80^{\circ} \mathrm{C}$ el comportamiento es similar a la de $60{ }^{\circ} \mathrm{C}$, el inconveniente encontrado es que la precipitación del uranio es menor para $80^{\circ} \mathrm{C}$ que para $25^{\circ} \mathrm{C}$.

De las tres condiciones de $\mathrm{pH}$ se obtuvo que el $\mathrm{pH}$ de precipitación óptimo se da para el valor de 2,5, siendo coincidente con la Bibliografía de MORAIS et al [3]. Sin embargo para $\mathrm{pH}$ superiores la respuesta también es buena solo que los valores alcanzados en precipitación de uranio como $\mathrm{UO}_{4}{ }_{2} \mathrm{H}_{2} \mathrm{O}$ no son los esperados.

Es importante destacar que para las temperaturas de $60^{\circ} \mathrm{C}$ y $80^{\circ} \mathrm{C}$ es muy importante el agregado en exceso de $\mathrm{H}_{2} \mathrm{O}_{2}$ dado que durante el calentamiento se produce evaporaciones del reactivo.

Planteando los cálculos estequeométricos se obtuvo que la concentración óptima del reactivo oxidante $\mathrm{H}_{2} \mathrm{O}_{2}$ supera el $30 \%$ en exceso a la estequiometria es decir para $0,9 \mathrm{~g}$, para las tres condiciones diferente de $\mathrm{pH}$ y temperatura.

La acidificación se obtuvo con el agregado de $\mathrm{HNO}_{3}$ el consumo del mismo se obtuvo teniendo en cuenta los cálculos estequeométricos con respecto al carbonato presente, según lo demostró FERNANDEZ et al [4].

Para el mecanismo de precipitación con la solución de NaF se obtuvieron las condiciones de concentración óptimas para las cuales el porcentaje de uranio en la solución es menor, siendo concentración de la solución del $\mathrm{NaF}$ 0,5M, tiempo de contacto 12 hrs y sin agitación generando un compuesto insoluble como es el $\mathrm{NaUF}_{6}$. Otros trabajos estudiados como es VIGIER et al [5] utilizan condiciones redox y altas temperaturas, pero los costos y las variables operativas del proceso de precipitación aumentan considerablemente.

Los valores del porcentaje de uranio en solución obtenidos del segundo mecanismo son mayores con respecto al primero, por lo cual se puede inferir que la respuesta para la precipitación de uranio fue mejor para el agente precipitante como es el $\mathrm{H}_{2} \mathrm{O}_{2}$ obteniendo el $\mathrm{UO}_{4} 2 \mathrm{H}_{2} \mathrm{O}$ precipitado.

\section{CONCLUSIONES}

En este trabajo se concluye que los rendimientos obtenidos con el primer método de precipitación son francamente satisfactorios para las condiciones operacionales de trabajo que se resumen en $\mathrm{pH}: 2,5$; temperatura de $60{ }^{\circ} \mathrm{C}$ y tiempos de reacción superiores a $3 \mathrm{~h}$ hasta $4 \mathrm{~h}$. Estas condiciones se determinaron analizando los valores obtenidos durante la precipitación de soluciones para la estructura química del uranio de $\mathrm{UO}_{2} * 2 \mathrm{H}_{2} \mathrm{O}$, planteada en la ecuación 3. No sucediendo lo mismo para el segundo mecanismo de precipitación, las respuestas no fueron satisfactorias, según se plantea en la ecuación química 4.

Lo más importante es obtener una buena precipitación del uranio con la determinación de la concentración óptima del consumo del agentes oxidante $\mathrm{H}_{2} \mathrm{O}_{2}$ para el cual se logra la mayor recuperación de $\mathrm{UO}_{2} * 2 \mathrm{H}_{2} \mathrm{O}$. La óptima concentración de trabajo obtenida para el $\mathrm{H}_{2} \mathrm{O}_{2}$ fue de $30 \%$ en exceso con respecto al valor estequeométrico calculado. 


\section{BIBLIOGRAFÍA}

[1] MILLER W. M., NEWELL STANNARD J.. "Environmental Toxicity of Aquatic Radionuclides: Models and Mechanisms", Ann Arbor Science Publishers, P.O. Box 1425, Ann Arbor, Michigan 48106. 1976.

[2] SMIRNOV A.L., SKRIPCHENKO S.Yu., RYCHKOVA V.N. , PASTUKHOV A.M., SHTUTSA M.G... "Uranium stripping from tri-n-butyl phosphate by hydrogen peroxide solutions", Revista Hydrometallurgy, v 137, pp 18-22. 2013.

[3] MORAIS C.A., GOMIERO L.A., SCASSIOTTIFILHO W. , RANGEL H. "Uranium stripping from tertiary amine by sulfuric acid solution and its precipitation as uranium peroxide", Revista Minerals EngineerIng, v 18. 2005. ISSN 13311333.

[4] FERNANDEZ R., BATUECAS RODRIGUEZ T., VERA PALOMINO J.. Ataque alcalino y beneficio de minerales del grupo de la uranita, Comisión general de energía atómica (CENEA), 1956.

[5] VIGIER J., LAPLACE A, RENARD C., MIGUIRDITCHIAN M., ABRAHAM F. "Uranium (III) precipitation in molten chloride by wet argon sparging", Journal of Nuclear Materials, n.474, pp 19-27, 2016.

[6] ELSHAFEEA H. Y. ABOW SLAMA, EBRAHEEM E., SAM A. K., "Precipitation and purification of uranium from rock phosphate", Journal of Radioanalytical and Nuclear Chemistry, v.299, n.1, pp 815-818. Enero 2014. ISSN: 0236-5731.

[7] SIDDIQUI I. A., SHAH B. V,. TADPHALE S. H AND KUMAR S. V. A continuous process for precipitation of ammonium diuranate from uranyl solution. Part I, Process Engineering and Systems Division, Government of India Atomic Energy Commission, 1988.

[8] WILFRED W. SCOTT, SC. D. D. Scott's Standard Methods of Chemical Analysis, Fifth Edition, v. I. Van Nostrand Company, Inc. Londres. 1945.

[9] HABASHI F. Handbook of Extractive Metallurgy (Wiley), v. 3, pp 1599-1648, 1997.

[10] U.S. ENVIRONMENTAL PROTECTION AGENCY. " Extraction and Beneficiation of Ores and Minerals. Uranium". Technical Resourse Document., v. 5. 1995.

[11] INTERNACIONAL ATOMIC ENERGY AGENCY. "Significance of mineralogy in the development of flowsheets for the processing of Uranium ores", Technical Report Series, n.196, 1980.

[12] INTERNACIONAL ATOMIC ENERGY AGENCY. "Uranium extraction technology", Technical Report Series, n. 359, Vienna, 1993. 